\title{
Eliminating microscopic lymph node metastasis by performing pelvic lymph node dissection during radical prostatectomy for prostate cancer
}

\author{
NOBUKI FURUBAYASHI ${ }^{1}$, TAKAHITO NEGISHI ${ }^{1}$, TOMOHARU UOZUMI ${ }^{1}$, KOUICHI SHIRAISHI ${ }^{1}$, \\ KENICHI TAGUCHI $^{2}$, MOTOTSUGU SHIMOKAWA ${ }^{3}$ and MOTONOBU NAKAMURA ${ }^{1}$ \\ Departments of ${ }^{1}$ Urology and ${ }^{2}$ Pathology; ${ }^{3}$ Institute for Clinical Research, \\ National Hospital Organization Kyushu Cancer Center, Fukuoka 811-1395, Japan
}

Received March 8, 2018; Accepted November 25, 2019

DOI: $10.3892 / \mathrm{mco} .2019 .1965$

\begin{abstract}
The oncological benefit of pelvic lymph node dissection (PLND) for prostate cancer (PCa) remains unclear. The therapeutic effect of PLND on the elimination of microscopic metastases during radical prostatectomy (RP) for $\mathrm{PCa}$ was examined in the current study. A total of 348 Japanese patients with high- or intermediate-risk PCa without lymph node metastasis, who underwent antegrade RP at the Kyushu Cancer Center (Fukuoka, Japan) between August 1998 and May 2013 were retrospectively analyzed. The patients were divided into the standard (obturator + internal iliac nodes) group and the expanded (standard + additional nodes) group according to the extent of PLND. Preoperative and postoperative characteristics were also analyzed to determine the factors associated with prostate-specific antigen (PSA) failure. Standard and expanded PLND were performed in $70.9 \%$ (247/348) and $29.1 \%$ (101/348) of cases, respectively. The results revealed that preoperative PSA levels were the only marked difference between the two groups. No differences were observed in the other preoperative and postoperative characteristics. Furthermore, the rate of PSA recurrence in each group did not differ to a statistically significant extent $(\mathrm{P}=0.3622)$. Reducing the area of dissection from expanded PLND to standard PLND significantly reduced the number of dissected lymph nodes $(\mathrm{P}<0.0001)$. Additionally, the PSA level, clinical tumor stage, Gleason score of the biopsy specimen, pathological tumor stage and extent of PLND were all associated with PSA recurrence, as determined via multivariate Cox hazards regression analysis $(\mathrm{P}=0.0177, \mathrm{P}=0.0023$,
\end{abstract}

Correspondence to: Dr Nobuki Furubayashi, Department of Urology, National Hospital Organization Kyushu Cancer Center, Notame 3-1-1, Minami-ku, Fukuoka 811-1395, Japan

E-mail: furubayashi-jua@umin.ac.jp

Key words: prostate cancer, radical prostatectomy, microscopic lymph node metastases, pelvic lymph node dissection, prostate-specific antigen failure
$\mathrm{P}=0.0027, \mathrm{P}<0.0001$ and $\mathrm{P}=0.0164$, respectively). In high- and intermediate-risk patients without lymph node metastasis, a greater number of lymph nodes were dissected when the extent of dissection was greater. Furthermore, the extent of PLND was a significantly associated with PSA failure. The results indicated that PLND exerted a therapeutic effect by eliminating microscopic pelvic lymph node metastases that were not detected by routine pathological examinations.

\section{Introduction}

Pelvic lymph node dissection (PLND) is the only reliable technique for accurately ensuring the nodal status in prostate cancer (PCa) (1-3), as the ability of imaging modalities such as computed tomography and standard magnetic resonance imaging to predict lymph node invasion is limited (4,5). Based on nomograms predicting the risk of preoperative lymph node metastasis, it is generally accepted that extended PLND is desirable in patients deemed suitable for PLND (6,7). Several studies have suggested that more extensive lymphadenectomy is associated with a survival advantage, possibly due to the elimination of microscopic metastases (8-11); however, there is no definitive proof of an oncologic benefit (12). The elimination of microscopic metastasis means that lymph node metastases that are not detected by routine pathological examinations are surgically removed by PLND. Thus, if the expanded PLND template is a factor influencing prostate-specific antigen (PSA) failure after radical prostatectomy (RP) in patients who are pathologically negative for lymph node metastasis, we might be able to indirectly detect the elimination of microscopic metastasis and confirm an improved therapeutic benefit over a more constrained template.

The aim of this study was to determine whether the extent of PLND is associated with the risk of PSA failure in patients undergoing RP, especially in high- and intermediate-risk PCa patients in whom lymph node metastasis is not detected.

\section{Patients and methods}

Patient characteristics and risk-group classification. The cases of 638 consecutive patients with clinically localized Pca 
who underwent RP at the Kyushu Cancer Center (Fukuoka, Japan) between August 1998 and May 2013 were reviewed. $\mathrm{RP}$ was performed in an open retropubic manner in all cases. The patients were classified into three risk groups according to the D'Amico criteria (13). A total of 290 patients were excluded from this study for the following reasons: A history of hormone therapy $(n=151)$, low-risk classification according to the D'Amico criteria ( $n=105)$, the absence of PLND $(n=14)$, the detection of lymph node metastasis by a routine pathological examinations [ $n=13$ (low-risk, $n=0$; intermediate-risk, $\mathrm{n}=5$; high-risk, $\mathrm{n}=8)]$ and unclear findings in the examination of biopsy or prostatectomy specimens $(n=7)$. Two pathologists evaluated the degree of malignancy in the biopsy and prostatectomy specimens and determined the pathological stage based on the 2009 tumor-node-metastasis classification (14).

PLND technique. At a minimum, all patients underwent standard PLND, which was performed along the lower edge of the external iliac vein with the caudal limit being the deep circumflex iliac vein and femoral canal, preserving the lymphatics overlying the external iliac artery. The proximal border was the bifurcation of the common iliac artery, and all tissue in the angle between the external and internal iliac arteries and obturator nerve was removed. All of the fatty, connective and lymphatic tissue of the obturator fossa was removed along the obturator muscle, leaving the obturator nerve and vessels bare. Subsequently, the internal iliac artery and the internal iliac vein (to the extent that was possible) were skeletonized up to the obturator arteriovenous branch. The patients were subdivided into two subgroups according to the lymph node dissection technique: Standard PLND and expanded (extended + more extended) PLND. Extended PLND included standard PLND as well as the dissection of the lymphatics overlying the external iliac artery and vein, this extended laterally to the genitofemoral nerve. More extended PLND included extended PLND as well as the dissection of the lymphatics overlying the common iliac artery, this extended cranially to the ureteric crossing.

Tissue processing and the determination of the PSA level. The RP and PLND specimens were fixed in $15 \%$ neutral buffered formalin (Wako Pure Chemical Industries, Ltd.) for 48-96 h at room temperature, and whole-organ prostate specimens were serially sectioned perpendicular to the rectal surface at $5-\mathrm{mm}$ intervals. Sections that were predominantly caudal and cephalic were cut at 5-mm intervals on the sagittal plane in order to assess the bladder neck and apical margins. The specimens were subsequently embedded in paraffin, cut into $5-\mu \mathrm{m}$ sections and stained with hematoxylin and eosin. Extraprostatic extension was defined as the extension of the tumor from the prostate to the periprostatic soft tissue. A positive resection margin was defined by the presence of tumor cells at the stained resection margin.

The follow-up schedule after RP included the performance of a PSA assay every three months for the first two years, followed by every four months for the next three years, and every six months thereafter. The date of disease recurrence or PSA failure was defined as the date on which a serum PSA level of $>0.2 \mathrm{ng} / \mathrm{ml}$ was detected. RP was performed if the PSA level did not drop below $0.2 \mathrm{ng} / \mathrm{ml}$ after surgery. Additional treatment is basically performed for cases meeting the criteria for PSA failure and adjuvant therapy is not routinely used in cases with a positive surgical margin or any unfavorable factors. A small number of patients who underwent RP were subsequently treated with radiotherapy and/or hormone therapy before the serum PSA level exceeded $0.2 \mathrm{ng} / \mathrm{ml}$. Thus, in these patients the date on which adjuvant therapy was initiated was defined as the date of disease recurrence. All patients provided their written informed consent to participate in this study, and the study protocol was approved by the Ethics Committee of the Kyushu Cancer Center.

Statistical analyses. All statistical analyses were performed using the JMP ${ }^{\circledR}$ Pro, version 13.0.0, software package (SAS Institute, Inc.). The PSA failure-free rate was determined according to the Kaplan-Meier method, and the significance of the clinicopathological parameters associated with PSA failure was assessed using the Cox proportional hazards regression model. Chi-squared and Mann-Whitney U tests were used to assess the differences between standard PLND and expanded PLND. P-values of $<0.05$ were considered to indicate statistical significance.

\section{Results}

Clinicopathological characteristics. The clinicopathological characteristics according to the PLND technique that was applied are presented in Table I. All patients were Japanese (median age, 66 years; range, 48-77), and the median PSA level was $8.171 \mathrm{ng} / \mathrm{ml}$ (range, 0.8 to $39.413 \mathrm{ng} / \mathrm{ml}$; normal range $<4.0 \mathrm{ng} / \mathrm{ml}$ ). The median follow-up period after surgery was 53.7 months. The standard PLND group included 247 (70.9\%) patients, while the expanded PLND group included $101(29.1 \%)$ patients. There were no marked differences in the preoperative characteristics of the two groups, including age, clinical tumor stage, and the Gleason score of the biopsy specimen (Table I). However, there was a significant difference in the preoperative PSA level $(\mathrm{P}=0.0008)$. There were no marked differences in the postoperative characteristics, including the pathological tumor stage, final Gleason score, extraprostatic extension, resection margin, and seminal vesicle invasion, of the two groups. The rate of PSA recurrence in the two groups did not differ to a statistically significant extent.

Association between the number of dissected lymph nodes and the PLND technique. In total, 247 patients (70.9\%) underwent standard PLND, and 101 patients (29.1\%) underwent expanded PLND (extended, $\mathrm{n}=78$; more extended, $\mathrm{n}=23$ ). The median number of dissected lymph nodes in the standard PLND group was 13, while that in the expanded PLND group was 19; the difference was statistically significant $(\mathrm{P}<0.0001$; Table II).

Associations between the patient characteristics and PSA failure. In the Cox proportional hazards analysis, all characteristics without a preoperative variable (i.e., age), were found to be significant predictors in the univariate analysis (Table III). The multivariate analysis revealed significant differences 
Table I. Clinicopathological characteristics according to the pelvic lymph node dissection technique.

\begin{tabular}{|c|c|c|c|c|c|}
\hline \multirow[b]{2}{*}{ Variable } & \multirow{2}{*}{$\begin{array}{c}\text { Standard } \\
\text { Standard } \\
\text { (obturator }+ \\
\text { internal iliac) }\end{array}$} & \multirow{2}{*}{$\begin{array}{c}\text { Expanded } \\
\text { Extended }+ \\
\text { more extended } \\
(\text { standard }+\alpha)\end{array}$} & \multirow[b]{2}{*}{ P-value } & \multicolumn{2}{|c|}{ Expanded } \\
\hline & & & & $\begin{array}{c}\text { Extended } \\
\text { (standard + } \\
\text { external iliac) }\end{array}$ & $\begin{array}{c}\text { More extended } \\
\text { (standard + external } \\
\text { and commom iliac) }\end{array}$ \\
\hline Total number of patients, $\mathrm{n}(\%)$ & 247 (70.9) & $101(29.1)$ & & 78 & 23 \\
\hline \multicolumn{6}{|l|}{ Age, n (\%) } \\
\hline$<70$ years & $166(67.2)$ & $69(68.3)$ & 0.8407 & $58(74.4)$ & $11(47.8)$ \\
\hline$\geq 70$ years & $81(23.8)$ & $32(31.7)$ & & $20(25.6)$ & $12(52.2)$ \\
\hline \multicolumn{6}{|l|}{ Preoperative PSA, n (\%) } \\
\hline$\leq 10$ & $170(68.8)$ & $50(49.5)$ & 0.0008 & $40(51.3)$ & $10(43.5)$ \\
\hline$>10$ & $77(31.2)$ & $51(50.5)$ & & $38(48.7)$ & $13(56.5)$ \\
\hline \multicolumn{6}{|l|}{ Clinical T stage, $\mathrm{n}(\%)$} \\
\hline cT1c & $155(62.8)$ & $72(71.3)$ & 0.1256 & $54(69.2)$ & $18(78.3)$ \\
\hline$\geq \mathrm{cT} 2 \mathrm{a}$ & $92(37.2)$ & $29(28.7)$ & & $24(30.8)$ & $5(21.7)$ \\
\hline \multicolumn{6}{|l|}{ Biopsy Gleason score, n (\%) } \\
\hline$\leq 7$ & $171(69.2)$ & $70(69.3)$ & 0.9888 & $54(69.2)$ & $16(69.6)$ \\
\hline$>8$ & $76(30.8)$ & $31(30.7)$ & & $24(30.8)$ & $7(30.4)$ \\
\hline \multicolumn{6}{|l|}{ Pathological T stage, n (\%) } \\
\hline$\leq \mathrm{pT} 2$ & $147(59.5)$ & $57(56.4)$ & 0.5972 & $43(55.1)$ & $14(60.9)$ \\
\hline$\geq \mathrm{pT} 3$ & $100(40.5)$ & $44(43.6)$ & & $35(44.9)$ & $9(39.1)$ \\
\hline \multicolumn{6}{|l|}{ Final Gleason score, n (\%) } \\
\hline$\leq 7$ & $189(76.5)$ & $71(70.3)$ & 0.2303 & $55(70.5)$ & $17(73.9)$ \\
\hline$>8$ & $58(23.5)$ & $30(29.7)$ & & $23(29.5)$ & $6(26.1)$ \\
\hline \multicolumn{6}{|l|}{ Extraprostatic extension, $\mathrm{n}(\%)$} \\
\hline 0 & $167(67.6)$ & $64(63.4)$ & 0.4486 & $50(64.1)$ & $14(60.9)$ \\
\hline 1 & $80(32.4)$ & $37(36.6)$ & & $28(35.9)$ & $9(39.1)$ \\
\hline \multicolumn{6}{|l|}{ Resection margin, n (\%) } \\
\hline 0 & $189(76.5)$ & $84(83.2)$ & 0.1632 & $64(82.1)$ & $20(86.9)$ \\
\hline 1 & $58(23.5)$ & $17(16.8)$ & & $14(17.9)$ & $3(13.1)$ \\
\hline \multicolumn{6}{|l|}{ Seminal vesicle invasion, $\mathrm{n}(\%)$} \\
\hline 0 & $231(93.5)$ & $98(97.0)$ & 0.1668 & $75(96.2)$ & $23(100)$ \\
\hline 1 & $16(6.5)$ & $3(3.0)$ & & $3(3.8)$ & 0 \\
\hline PSA resurrence, n (\%) & $44(17.8)$ & $14(13.9)$ & 0.3622 & $12(15.4)$ & $2(8.7)$ \\
\hline
\end{tabular}

PSA, prostate-specific antigen.

between the patients with and without PSA failure in the preoperative PSA level, clinical tumor stage, and Gleason score of the biopsy specimen (preoperative characteristics) and in the pathological tumor stage and extent of PLND (postoperative characteristics) (Table III).

\section{Discussion}

Urological surgeons have been performing extended pelvic lymph node dissection in high- and intermediate-risk cases because several studies have suggested that more extensive lymphadenectomy is associated with a survival advantage, possibly due to the elimination of microscopic metastasis (8-11). However, a systematic review of studies assessing the relative benefit and harm of PLND in relation to the oncological and non-oncological outcomes of patients undergoing RP for PCa failed to confirm a direct therapeutic effect. The current poor quality of evidence indicates the need for robust and adequately powered clinical trials (12). Thus, the therapeutic role of PLND during radical prostatectomy for the management of PCa remains controversial. The patients in the low-risk group were presumed to have a minimal risk of developing lymph node metastasis $(6,7,15)$. Thus, the therapeutic role of PLND in low-risk patients is not clear. The overall results of RP operations performed in our institution showed that no low-risk patients developed lymph node metastasis. Thus, low-risk patients were excluded from the present study, which focused on intermediate- and high-risk patients. In the 
Table II. Association between lymph node dissection number and the pelvic lymph node dissection technique.

\begin{tabular}{|c|c|c|c|c|c|}
\hline \multirow[b]{2}{*}{ Variable } & \multirow{2}{*}{$\begin{array}{c}\text { Standard } \\
\text { Standard } \\
\text { (obturator }+ \\
\text { internal iliac) }\end{array}$} & \multirow{2}{*}{$\begin{array}{c}\text { Expanded } \\
\text { Extended }+ \text { more } \\
\text { extended } \\
(\text { standard }+\alpha)\end{array}$} & \multirow[b]{2}{*}{$\mathrm{P}$-value } & \multicolumn{2}{|c|}{ Expanded } \\
\hline & & & & $\begin{array}{l}\text { Extended (standard } \\
+ \text { external iliac) }\end{array}$ & $\begin{array}{c}\text { More extended } \\
\text { (standard + external and } \\
\text { commom iliac) }\end{array}$ \\
\hline Total number of patients, n (\%) & $247(70.9)$ & $101(29.1)$ & - & 78 & 23 \\
\hline $\begin{array}{l}\text { Total number of dissected nodes, } \\
\text { median (range) }\end{array}$ & $13(0-31)$ & $19(5-40)$ & $<0.0001$ & $19(5-40)$ & $19(13-35)$ \\
\hline Upper obturator & $10(0-29)$ & $8(2-21)$ & - & $9(2-21)$ & $8(2-18)$ \\
\hline Downer obturator + internal & $2(0-14)$ & $2(0-16)$ & & $2(0-11)$ & $1(0-16)$ \\
\hline External iliac & - & $7(0-19)$ & & $7(0-19)$ & $8(2-15)$ \\
\hline Common iliac & - & $0(0-8)$ & & - & $2(0-8)$ \\
\hline
\end{tabular}

Table III. Association between patient characteristics and PSA failure.

\begin{tabular}{|c|c|c|c|}
\hline \multicolumn{4}{|l|}{ A, Univariate analysis } \\
\hline Characteristics & Hazard ratio & $95 \% \mathrm{CI}$ & P-value \\
\hline Age, $<70$ vs. $\geq 70$ years & 1.275 & $0.739-2.150$ & 0.3745 \\
\hline Preoperative PSA, $\leq 10$ vs. $>10$ & 2.333 & $1.391-3.936$ & 0.0014 \\
\hline Clinical T stage, cT1c vs. $\geq c \mathrm{~T} 2 \mathrm{a}$ & 3.268 & $1.941-5.609$ & $<0.0001$ \\
\hline Biopsy Gleason score $\leq 7$ vs. $>8$ & 3.005 & $1.792-5.069$ & $<0.0001$ \\
\hline Pathological T stage, $\leq$ pT2 vs. $\geq$ pT3 & 5.267 & $2.962-9.973$ & $<0.0001$ \\
\hline Final Gleason score $\leq 7$ vs. $>8$ & 2.986 & $1.775-5.007$ & $<0.0001$ \\
\hline Extraprostatic extension & 3.608 & $2.139-6.225$ & $<0.0001$ \\
\hline Resection margin & 2.578 & $1.493-4.353$ & 0.0009 \\
\hline Seminal vesicle invasion & 4.465 & $2.042-8.690$ & 0.0005 \\
\hline Range of PLND, standard vs. expanded & 1.827 & $1.009-3.523$ & 0.0466 \\
\hline
\end{tabular}

B, Multivariate analysis

\begin{tabular}{lrrr}
\hline Preoperative PSA, $\leq 10$ vs. $>10$ & 1.934 & $1.122-3.344$ & 0.0177 \\
Clinical T stage, cT1c vs. $\geq$ cT2a & 2.293 & $1.343-3.983$ & 0.0023 \\
Biopsy Gleason score $\leq 7$ vs. $>8$ & 2.260 & $1.329-3.863$ & 0.0027 \\
Pathological T stage, $\leq$ pT2 vs. $\geq$ pT3 & 4.004 & $2.199-7.717$ & $<0.0001$ \\
Final Gleason score $\leq 7$ vs. $>8$ & 1.387 & $0.742-2.583$ & 0.3038 \\
Extraprostatic extension & 0.864 & $0.419-2.011$ & 0.7141 \\
Resection margin & 1.549 & $0.834-2.846$ & 0.1638 \\
Seminal vesicle invasion & 1.749 & $0.779-3.542$ & 0.1643 \\
Range of PLND, standard vs. expanded & 2.099 & $1.140-4.099$ & 0.0164
\end{tabular}

Clinical and pathological staging was based on the TNM classification (2009). PSA, prostate-specific antigen; CI, confidence interval; PLND, pelvic lymph node dissection.

seminal article describing the natural history of biochemical recurrence after radical prostatectomy, Pound et al reported that the median actuarial time from biochemical recurrence to metastasis was 8 years. Furthermore, the median actuarial time to death was 5 years, although this depended on the time from biochemical recurrence to metastasis (16). Makarov et al reported that the median (range) time to failure (as estimated by the Kaplan-Meier method) was 24 months (12 to 144 months) from radical prostatectomy to PSA failure, 36 months (0 to 132 months) from PSA failure to metastasis, 
84 months (12 to 180 months) from metastasis to death and 168 months (24 to 216 months) from radical prostatectomy to death (17). During a median follow-up period of 53.7 months, no patients died of prostate cancer ( 8 patients died of other disease). This median follow-up period was considered too short to evaluate the oncologic outcomes based on OS. The oncologic outcome was therefore assessed based on PSA failure. Additional treatment is basically performed for cases that meet the criteria of PSA failure, and it is very rare to detect recurrent lesions by imaging modalities such as computed tomography and standard magnetic resonance imaging at this point. The purpose of the current study was to clarify the therapeutic effect of PLND by investigating whether or not the extent of PLND is associated with the risk of PSA failure in high- and intermediate-risk PCa patients without lymph node metastasis.

In the present study, the high- and intermediate-risk PCa patients without lymph node metastasis were classified into two groups according to the extent of PLND: Standard PLND (obturator + internal iliac) and expanded PLND (standard + additional nodes), accounting for $70.9 \%$ (247/348) and $29.1 \%(101 / 348)$ of the patients, respectively (Table I). The preoperative PSA level was the only value for which a marked difference was observed between the two groups; no significant differences were observed in the other preoperative and postoperative characteristics. In addition, there was no marked difference in the rate of PSA recurrence between the groups $(\mathrm{P}=0.3622)$. The extent of PLND varies widely according to the era, institution, operative procedure and individual urologist due to the lack of standardized definitions regarding anatomical extension $(16,17)$. The definition of extended PLND differs according to the guidelines used. According to the National Comprehensive Cancer Network (NCCN) guidelines, extended PLND includes the removal of all node-bearing tissue from the area bounded by the external iliac vein (anteriorly), the pelvic side wall (laterally), the bladder wall (medially), the floor of the pelvic (posteriorly), Cooper's ligament (distally) and the internal iliac artery (proximally) (6). In contrast, according to the European Association of Urology (EAU), extended PLND includes the removal of the nodes overlying the external iliac artery and vein, the nodes within the obturator fossa, located cranially and caudally to the obturator nerve, and the nodes located medially and laterally to the internal iliac artery (7). The major difference between these guidelines concerns the definition of extended PLND-specifically whether or not the nodes overlying the external iliac artery are resected. At the Kyushu Cancer Center, the extent of standard PLND is similar-but not identical-to the definition of extended PLND in the NCCN guidelines, as it was performed along the lower edge of the external iliac vein, without the resection of the nodes overlying the external iliac vein.

When RP was initially performed at our institution, all cases underwent more extended PLND, which includes the common iliac, external, obturator and internal lymph nodes, as there was no consensus among urological surgeons regarding the extent of PLND, and because the performance of PLND with an increased range may allow for the surgical resection of microscopic lymph node metastasis. However, even when patients who received preoperative hormone therapy were included, the rates of lymph node metastasis and PSA recurrence after $\mathrm{RP}$ at the Kyushu Cancer Center were lower in comparison to previous studies $(18,19)$. Thus, as more RP procedures were performed, the extent of lymph node dissection was gradually reduced to extended PLND and finally standard PLND, regardless of the D'Amico risk classification. The advantage of this single-institutional study over a multicenter study is that all of the operations were performed by or under the supervision of urological surgeons, who performed standardized surgery. For this reason, any differences in the dissection area and methods were negligible. There are no established guidelines regarding the optimum method of examining PLND specimens, and the approach may vary considerably between individual pathologists and institutions (20). At our institution, PLND specimens were processed using the same methods as the RP specimens. Although the period was long, this study was performed in a single institution and the tissue processing and diagnostic methods basically remained unchanged during the study period.

We also examined the number of lymph nodes dissected for each PLND technique (Table II). When the area of dissection was reduced by changing from expanded PLND to standard PLND, there was a significant decrease in the number of dissected lymph nodes $(\mathrm{P}<0.0001)$. Thus, as expected, the number of dissected lymph nodes was decreased due to the reduction in the extent of lymph node dissection. This also suggests that narrowing the extent of lymph node dissection might reduce the likelihood of eliminating microscopic lymph node metastasis by surgery.

To confirm the therapeutic effect of PLND, we next examined the correlation between patient characteristics and PSA failure in consecutive RP cases (Table III). With the exception of age, all factors were found to affect PSA recurrence in the univariate analysis. Ultimately, the PSA level, clinical tumor stage, Gleason score of the biopsy specimen, pathological tumor stage, and extent of PLND were found to affect the incidence of PSA recurrence in the multivariate analysis. These results suggest that PLND has a therapeutic effect because microscopic lymph node metastasis can be eliminated by PLND. Some studies have reported the possible effect of lymphadenectomy on the survival of patients with confirmed positive nodes who underwent radical prostatectomy. Bader et al reported a $78 \%$ cause-specific survival rate in patients treated with RP and ePLND and who did not undergo any adjuvant therapy until progression. Interestingly, among the patients with 1 positive node, $39 \%$ remained free of clinical or biochemical progression, in comparison to $12 \%$ of patients with 2 or more positive nodes (9). Seiler et al reported that patients with 1 positive node have a good survival probability and a $20 \%$ chance of remaining biochemical relapse-free after a median follow-up period of 15.6 years, even without immediate adjuvant therapy (21). It is considered that these reports apply to cases of micrometastasis. Yuen et al reported that sentinel lymph nodes were located in the obturator fossa, internal and external iliac regions, and rarely in the common iliac and presacral regions (22). There is a possibility that several LNs in the external iliac and common iliac area determined PSA recurrence. Two prospective studies (NCT01812902 and NCT01555086) are ongoing to determine the therapeutic effectiveness of PLND 
in terms of oncological outcomes. These results may improve the level of evidence (12). In the previous study, we reviewed all cases, including a low-risk group, and concluded that standard PLND is appropriate at radical prostatectomy (23). In this study, we reviewed the cases in the intermediate- and high-risk groups, and excluded cases in the low-risk group. Extended PLND is generally recommended for intermediateand high-risk patients. Thus, while the results of this study differed from those of previous studies, we do not consider it to be a problem.

The present study was associated with several limitations, including the small cohort size and the retrospective nature of our database analysis.

In conclusion, the extent of PLND in operations performed at the Kyushu Cancer Center has gradually been reduced over time, and standard PLND is routinely performed. In cases involving high- and intermediate-risk PCa patients without lymph node metastasis, a greater number of lymph nodes can be dissected when the extent of dissection is larger, and the extent of lymph node dissection was found to significantly affect PSA failure. Thus, we demonstrated that PLND exerts a therapeutic effect in intermediate- and high-risk patients by eliminating microscopic pelvic lymph node metastasis that is not detected by routine pathological examinations.

\section{Acknowledgements}

Not applicable.

\section{Funding}

No funding was received.

\section{Availability of data and materials}

The datasets used and/or analyzed during the current study are available from the corresponding author on reasonable request.

\section{Authors' contributions}

NF, TN and MN conceived and designed the present study. NF drafted the manuscript. TU, KS, KT and MS analyzed and interpreted the data. TN, TU, KS and MN interpreted the data and revised the manuscript critically for important intellectual content. All authors were involved in data interpretation and drafting the manuscript and have read and approved the final version of the manuscript.

\section{Ethics approval and consent to participate}

The present study was approved by the Institutional Review Board of the National Hospital Organization Kyushu Cancer Center (approval no. 2014-99). Written informed consent was obtained from the patient.

\section{Patient consent for publication}

The patient provided written informed consent for the publication of any associated data.

\section{Competing interests}

The authors declare that they have no competing interests.

\section{References}

1. Heidenreich A, Bellmunt J, Bolla M, Joniau S, Mason M, Matveev V, Mottet N, Schmid HP, van der Kwast T, Wiegel T, et al: EAU guidelines on prostate cancer. Part 1: Screening, diagnosis, and treatment of clinically localised disease. Eur Urol 59: 61-71, 2011

2. Briganti A, Blute ML, Eastham JH, Graefen M, Heidenreich A, Karnes JR, Montorsi F and Studer UE: Pelvic lymph node dissection in prostate cancer. Eur Urol 55: 1251-1265, 2009.

3. Van den Bergh L, Lerut E, Haustermans K, Deroose CM, Oyen R, Isebaert S, Budiharto T, Ameye F, Mottaghy FM, Bogaerts K, et al: Final analysis of a prospective trial on functional imaging for nodal staging in patients with prostate cancer at high risk for lymph node involvement. Urol Oncol 33: 109. e23-31, 2015.

4. Hövels AM, Heesakkers RA, Adang EM, Jager GJ, Strum S, Hoogeveen YL, Severens JL and Barentsz JO: The diagnostic accuracy of $\mathrm{CT}$ and MRI in the staging of pelvic lymph nodes in patients with prostate cancer: A meta-analysis. Clin Radiol 63: 387-395, 2008.

5. Briganti A, Abdollah F, Nini A, Suardi N, Gallina A, Capitanio U, Bianchi M, Tutolo M, Passoni NM, Salonia A, et al: Performance characteristics of computed tomography in detecting lymph node metastases in contemporary patients with prostate cancer treated with extended pelvic lymph node dissection. Eur Urol 61: 1132-1138, 2012.

6. National Comprehensive Cancer Network: Guidelines on prostate cancer. https://www.nccn.org/professionals/physician_gls/ pdf/prostate.pdf. Accessed February 1, 2018.

7. European Association of Urology: Guidelines on prostate cancer. https://uroweb.org/guideline/prostate-cancer/\#6. Accessed February 1, 2018.

8. Joslyn SA and Konety BR: Impact of extent of lymphadenectomy on survival after radical prostatectomy for prostate cancer. Urology 68: 121-125, 2006.

9. Bader P, Burkhard FC, Markwalder R and Studer UE: Disease progression and survival of patients with positive lymph nodes after radical prostatectomy. Is there a chance of cure? J Urol 169: 849-854, 2003

10. Daneshmand S, Quek ML, Stein JP, Lieskovsky G, Cai J, Pinski J, Skinner EC and Skinner DG: Prognosis of patients with lymph node positive prostate cancer following radical prostatectomy: Long-term results. J Urol 172: 2252-2255, 2004.

11. Wagner M, Sokoloff M and Daneshmand S: The role of pelvic lymphadenectomy for prostate cancer-therapeutic? J Urol 179: 408-413, 2008.

12. Fossati N, Willemse PM, Van den Broeck T, van den Bergh RCN, Yuan CY, Briers E, Bellmunt J, Bolla M, Cornford P, De Santis M, et al: The benefits and harms of different extents of lymph node dissection during radical prostatectomy for prostate cancer: A systematic review. Eur Urol 72: 84-109, 2017.

13. D'Amico AV, Whittington R, Malkowicz SB, Schultz D, Blank K, Broderick GA, Tomaszewski JE, Renshaw AA, Kaplan I, Beard CJ and Wein A: Biochemical outcome after radical prostatectomy, external beam radiation therapy, or interstitial radiation therapy for clinically localized prostate cancer. JAMA 280: 969-974, 1998.

14. Sobin LH, Gospodarowicz MK and Wittekind Ch: TNM Classification of Malignant Tumors. 7th edition. Wiley Blackwell, Oxford, 2009.

15. Mitsuzuka K, Koie T, Narita S, Kaiho Y, Yoneyama T, Kawamura S, Tochigi T, Ohyama C, Habuchi T and Arai Y: Is pelvic lymph node dissection required at radical prostatectomy for low-risk prostate cancer? Int J Urol 20: 1092-1096, 2013.

16. Pound CR, Partin AW, Eisenberger MA, Chan DW, Pearson JD and Walsh PC: Natural history of progression after PSA elevation following radical prostatectomy. JAMA 281: 1591-1597, 1999.

17. Makarov DV, Humphreys EB, Mangold LA, Carducci MA, Partin AW, Eisenberger MA, Walsh PC and Trock BJ: The natural history of men treated with deferred androgen deprivation therapy in whom metastatic prostate cancer developed following radical prostatectomy. J Urol 179: 156-162, 2008. 
18. Heidenreich A, Bastian PJ, Bellmunt J, Bolla M, Joniau S, van der Kwast T, Mason M, Matveev V, Wiegel T, Zattoni F, et al: EAU guidelines on prostate cancer. Part 1: Screening, diagnosis, and local treatment with curative intent-update 2013. Eur Urol 65: 124-137, 2014

19. Briganti A, Suardi N, Gallina A, Abdollah F and Montorsi F: Pelvic lymph node dissection in prostate cancer: The mystery is taking shape. Eur Urol 63: 459-461, 2013.

20. Prendeville $\mathrm{S}$ and van der Kwast TH: Lymph node staging in prostate cancer: Perspective for the pathologist. J Clin Pathol 69: 1039-1045, 2016

21. Seiler R, Studer UE, Tschan K, Bader P and Burkhard FC: Removal of limited nodal disease in patients undergoing radical prostatectomy: Long-term results confirm a chance for cure. J Urol 191: 1280-1285, 2014.
22. Yuen K, Miura T, Sakai I, Kiyosue A and Yamashita M: Intraoperative fluorescence imaging for detection of sentinel lymph nodes and lymphatic vessels during open prostatectomy using indocyanine green. J Urol 194: 371-377, 2015.

23. Furubayashi N, Negishi T, Iwai H, Nagase K, Taguchi K, Shimokawa $\mathrm{M}$ and Nakamura $\mathrm{M}$ : Determination of adequate pelvic lymph node dissection range for Japanese males, undergoing radical prostatectomy. Mol Clin Oncol 6: 775-781, 2017.

This work is licensed under a Creative Commons Attribution-NonCommercial-NoDerivatives 4.0 International (CC BY-NC-ND 4.0) License. 